\title{
The progenitors of magnetic white dwarfs in open clusters
}

\author{
B. Külebi ${ }^{1,2,3}$, J. Kalirai ${ }^{4,5}$, S. Jordan ${ }^{3}$, and F. Euchner ${ }^{6}$ \\ 1 Institut de Ciències de l'Espai (CSIC-IEEC), Facultat de Ciències, Campus UAB, Torre C5-parell, 2a planta, \\ 08193 Bellaterra, Spain \\ e-mail: bkulebi@ieec.cat \\ 2 Institute for Space Studies of Catalonia (ICE), c/Gran Capità 2-4, Edif. Nexus 104, 08034 Barcelona, Spain \\ 3 Astronomisches Rechen-Institut, Zentrum für Astronomie der Universität Heidelberg, Mönchhofstr. 12-14, 69120 Heidelberg, \\ Germany \\ ${ }^{4}$ Space Telescope Science Institute, 3700 San Martin Drive, Baltimore, MD 21042, USA \\ 5 Center for Astrophysical Sciences, Johns Hopkins University, Baltimore, MD 21218, USA \\ ${ }^{6}$ Swiss Seismological Service, ETH Zurich, Sonneggstrasse 5, 8092 Zurich, Switzerland
}

Received 18 January 2012 / Accepted 24 April 2013

\begin{abstract}
Context. White dwarfs are the final stages of stellar evolution for most stars in the galaxy and magnetic white dwarfs (MWDs) represent at least ten percent of the whole sample. According to the fossil-field hypothesis magnetic fields are remnants of the previous stages of evolution. However, population synthesis calculations are unable to reproduce the MWD sample without binary interaction or inclusion of a population of progenitor with unobservable small-scale fields.

Aims. One necessary ingredient in population synthesis is the initial-to-final-mass relation (IFMR) which describes the mass-loss processes during the stellar evolution. When white dwarfs are members of open clusters, their evolutionary histories can be assessed through the use of cluster properties. This enables an independent way of determining the mass of their progenitors. The discovery of the magnetic WD 0836+201 in the Praesepe cluster prompted the question whether magnetic fields affect the IFMR. In this work we investigate this suggestion through investigations of all three known MWDs in open clusters.

Methods. We assess the cluster membership by correlating the proper-motion of MWDs with the cluster proper-motion and by analyzing the candidates spectroscopically with our magnetic model spectra in order to estimate the effective temperature and radii. Furthermore, we use mass-radius relations and evolutionary models to constrain the histories of the probable cluster members.

Results. We identified SDSS J085523.87+164059.0 to be a proper-motion member of Praesepe. We also included the data of the formerly identified cluster members NGC 6819-8, WD 0836+201 and estimated the mass, cooling age and the progenitor masses of the three probable MWD members of open clusters. According to our analysis, the newly identified cluster member SDSS J085523.87+164059.0 is an ultra-massive MWD of mass $1.12 \pm 0.11 M_{\odot}$.

Conclusions. We increase the sample of MWDs with known progenitor masses to ten, with the rest of the data coming from the common proper motion binaries. Our investigations show that, when effects of the magnetic fields are included in the diagnostics, the estimated properties of these cluster MWDs do not show evidence for deviations from the IFMR. Furthermore we estimate the precision of the magnetic diagnostics which would be necessary to determine quantitatively whether magnetism has any effect on the mass-loss.
\end{abstract}

Key words. stars: magnetic field - stars: distances - open clusters and associations: general - stars: fundamental parameters white dwarfs

\section{Introduction}

White dwarfs are the final products of stellar evolution for more than $95 \%$ of the stars in our galaxy. One of the most important tools for the understanding of stellar evolution is the initial-tofinal-mass relation (IFMR), which, links the progenitor mass of a star to the final white dwarf mass (Weidemann 1977). The IFMR is inherently connected to mass-loss mechanisms (see e.g. Weidemann 2000). The understanding of stellar evolution from the perspective of the IFMR enables us to constrain the critical mass necessary for Type II supernova explosions. Along with the initial mass function, this information can be used to estimate the birthrates and energetics of the supernovae, as well as the birthrates of the SN II products, the neutron stars (van den Bergh \& Tammann 1991). The precise IFMR also constrains the galactic chemical evolution (i.e. enrichment in the interstellar medium), which in turn contributes to our understanding of the star formation efficiency in galaxies (Somerville \& Primack 1999).

The first cluster IFMR studies were performed by Weidemann (1977); Koester \& Weidemann (1980). With now about 40 white dwarfs associated with 11 clusters the empirical information on the IFMR has more than doubled over the last few years (see Kalirai et al. 2009; Casewell et al. 2009; Dobbie et al. 2009; Williams et al. 2009, for a recent discussion of cluster IFMR data). The increase in the data not only established the support for the already estimated general trend, in which higher stellar masses yield more massive white dwarfs, but also resulted in an increase in the scatter. This spread is argued to be the result of differences in the host environments. Marigo (2001) argued that one of these effects is metallicity, for which metal-rich stars undergo heavier mass-loss and yield, on average, lighter white dwarfs. Kalirai et al. (2005) was first to show this to be likely through spectroscopic observations of white dwarfs 
in NGC 2099, and later Kalirai et al. (2007) demonstrated the possibility of this effect in NGC 6791.

Another physical process affecting the final mass of the white dwarf was proposed to be rotation. The fact that angular momentum acts as an extra pressure support against gravity inside a degenerate structure has been known since the works of Anand (1965); Roxburgh (1965); Ostriker (1966). Within the context of stellar evolution simple numerical methods show that this extra pressure causes the rotating degenerate core to expand and hence keep the maximum temperature lower than the carbon ignition temperature (Dominguez et al. 1996). This lower temperature enables the growth of the $\mathrm{C}-\mathrm{O}$ core in mass while avoiding carbon ignition. In this way ultra-massive (1.2-1.4 $\left.M_{\odot}\right)$ white dwarfs can be formed from progenitors with masses between 6-8 $M_{\odot}$.

Magnetic fields, alongside angular momentum, were also proposed to be an effective factor of the mass-loss, after the discovery of a magnetic white dwarf (MWD) WD 0836+201 as a probable member of the Praesepe cluster (EG59, Claver et al. 2001; Catalán et al. 2008a). Note that it was mislabeled in both works as explained in Casewell et al. (2009). Considering WD 0836+201 as a Praesepe member, and not taking the magnetic field into consideration, the estimated white dwarf mass was higher than expected for its progenitor mass. If magnetism is an important factor affecting the IFMR, this would have implications for the MWD population.

MWDs constitute at least $10 \%$ of the white dwarfs if observational biases are considered (Liebert et al. 2003; Kawka et al. 2007). The current known population of MWDs has been increased considerably by the Sloan Digital Sky Survey (SDSS) to about 220 objects (Gänsicke et al. 2002; Schmidt et al. 2003; Vanlandingham et al. 2005; Külebi et al. 2009). SDSS also dramatically increased the total known white dwarf population (see e.g. Kleinman et al. 2013, for Data Release 7) and recent studies indicate that the number of MWDs in the SDSS could be as large as 521 (Kepler et al. 2013). The origin of the magnetic fields of MWDs is still under question. The accepted picture is the fossilfield hypothesis, where the field strengths are reminiscent of an earlier stage of stellar evolution. Due to the conservation of flux, the field strengths are amplified by the contraction of the stellar core to a white dwarf. For the case of MWDs, chemically peculiar Ap and Bp stars were proposed as the progenitors (Angel et al. 1981).

One problem of the fossil-field hypothesis is the relatively massive nature of the MWDs (Liebert 1988; Vennes \& Kawka 2008). While the mean value of the masses of the MWDs is $0.78 M_{\odot}$ (Kawka et al. 2007), the mean mass of the non-magnetic white dwarf sample is $0.661 M_{\odot}$ (Gianninas et al. 2011). The hypothesis that this could be a result due to the impact of magnetism on the mass-loss was considered by Wickramasinghe \& Ferrario (2005) via population synthesis. Their conclusion was that the current number distribution and masses of high-field magnetic white dwarfs (HFMWDs, $B \geq 10^{6} \mathrm{G}$ ) are not generated by an inclusion of a modified IFMR, but rather by considering the contribution of about 10 percent of A/B stars with unobservable small scale magnetic fields.

The aim of this work is to analyze the MWDs in clusters with sophisticated magnetic models, in order to estimate the progenitor masses. This is undertaken by calculating the masses of the MWDs and their progenitors by using the additional information estimated due to their membership in open clusters, namely their distances and total evolutionary ages

Up to now, only two MWDs have been discovered as members of open clusters, one in Praesepe (WD 0836+201) and the other one in NGC 6819 (NGC 6819-8, Kalirai et al. 2008). Neither of them has been analyzed with magnetic models so far. In order to increase the statistical sample further, we correlate the properties of SDSS white dwarfs that show evidence of magnetic fields with the kinematic properties of open clusters in order to determine the possibility of their cluster membership. While we identified only SDSS J085523.87+164059.0 (SDSS J0855+1640) as a member of an open cluster, we also analyzed NGC 6819-8. Furthermore, in this work we discuss former investigations on the influence of magnetism on the IFMR, compare our results with previous studies, and evaluate the analysis employed.

In Sect. 2 we assess the cluster membership of the currently known SDSS MWDs in the literature through proper-motion information. Afterwards, we describe our methods to analyze the cluster MWDs in Sect. 3 and explain what the relevant uncertainties are. In Sect. 4 we apply our method to cluster MWDs and in Sect. 5 we discuss whether we can conclude on possible effects of the magnetism on the IFMR

\section{Cluster membership}

In our investigation we compared the astrometric properties of 137 SDSS hydrogen-rich (DA) MWDs (see Külebi et al. 2009, and references therein) with the properties of 520 open clusters from the Catalogue of Open Cluster Data (Kharchenko et al. 2005). In order to assess the membership probabilities of the white dwarfs based on their kinematics, we use the propermotions estimated by the SDSS (Munn et al. 2004). The propermotions of the MWDs were acquired from the CASJOBS SQL interface (Li \& Thakar 2008).

Our criterion for open cluster membership was through checking whether the MWD lie within the tidal radius of an open cluster (values taken from the catalogue of Piskunov et al. 2008) and whether the proper-motion of the MWD is within $3 \sigma$ of the cluster proper-motion. As a result we obtained only one new possible open cluster member SDSS J0855+1640 of the open cluster NGC 2632 (Praesepe), which is a nearby open cluster that has been the subject of many white dwarf and IFMR investigations (Luyten 1962; Eggen \& Greenstein 1965; Claver et al. 2001). Additionally with our calculations we recover the prototypical cluster member MWD, WD 0836+201.

Another constraint on possible membership is the position of the white dwarfs in the color-magnitude diagram (CMD) with respect to the cooling sequence expected for the cluster (see Kalirai et al. 2008). Since this method involves a prescription for the parameters we want to evaluate, e.g. deviation from the expected IFMR due to the effect of magnetism, we did not undertake such an analysis. Rather, the evolutionary constraints on the cluster membership come from the estimated cooling age of the white dwarf, i.e. the cooling age cannot be larger than the cluster age.

Finally the membership possibility of both SDSS J0855+ 1640 and WD 0836+201 can be assessed through considering the studies made on the WD populations within the open clusters. Both of these MWDs are possible members of Prasepe. The current number of white dwarfs in this cluster, confirmed by radial velocity observations of Casewell et al. (2009) is eight, including the known magnetic WD 0836+201. Compared to numerical simulations of the dynamics of open clusters (Portegies Zwart et al. 2001) the number of white dwarfs found in Praesepe is lower than expected, especially if compared to the number of observed giants. Hence it is logical to expect more 
Table 1. Photometric and astrometric properties of the DAHs with possible Praesepe memberships.

\begin{tabular}{lccccccc}
\hline \hline MWD & RA/(h m s) & Dec/(deg $\left.{ }^{\prime \prime}{ }^{\prime \prime}\right)$ & $r / \mathrm{mag}$ & $i / \mathrm{mag}$ & $z / \mathrm{mag}$ & RA p.m./(mas/yr) & Dec p.m./(mas/yr) \\
\hline WD 0836+201 & 083945.56 & +200015.7 & $18.11 \pm 0.01$ & $18.36 \pm 0.01$ & $18.66 \pm 0.04$ & $-32.93 \pm 2.87$ & $-15.98 \pm 2.87$ \\
SDSS J0855+1640 & 085523.87 & +164059.0 & $18.80 \pm 0.01$ & $19.05 \pm 0.02$ & $19.32 \pm 0.08$ & $-33.14 \pm 2.75$ & $-14.71 \pm 2.75$ \\
\hline
\end{tabular}

Notes. The columns indicate the right ascension (RA), declination (Dec); the SDSS photometric magnitudes $r, i, z$ which are relevant for this work; and finally the proper-motions. Compare the proper-motions of the objects with the proper-motions of Praesepe RA p.m. $=-35.90 \pm 0.13$ mas/yr, Dec p.m. $=-12.88 \pm 0.11 \mathrm{mas} / \mathrm{yr}$ (Kharchenko et al. 2005).

white dwarfs to be discovered if the search is extended at least up to the tidal radius, as has been done in our work.

\section{Analysis}

One of the necessary ingredients to determine the cooling age of a white dwarf is the determination of $T_{\text {eff }}$ and $\log g$ values. Although this is undertaken by $\chi^{2}$ fits to the spectral lines for the non-magnetic white dwarfs, the situation for the MWDs is more complicated due to the influence of magnetism on the atomic transitions and the distribution of the magnetic field strengths over their surface.

To overcome this difficulty and to analyze the magnetic field geometry of the MWDs, we use a two-step analysis approach in which first the model spectra for magnetized atmospheres are calculated for a distribution of magnetic field vectors with respect to the line of sight and the normal on the surface of the star (see Jordan 1992; Jordan \& Schmidt 2003). This is repeated for given $T_{\text {eff }}$ and $\log g$ values and a database is generated.

Later these spectra representing individual vectors could be accessed from the database and added up in order to calculate the spectrum for a given geometry. The parameters of the field geometry are determined by an evolutionary fitting procedure (see Euchner et al. 2002).

This approach was used by Külebi et al. (2009) where the models in terms of offset dipoles for the single phase of these white dwarfs were determined. However, for that work the $T_{\text {eff }}$ values were determined by the photometric colors. In this current work, we use the same database and procedure to determine the $T_{\text {eff }}$ value of the MWDs in detail.

Unfortunately, for MWDs it is not possible to determine the $\log g$ values due to the difficulties in accounting for the simultaneous impact of Stark and Zeeman effects on the spectral lines. Moreover, no atomic data exists for hydrogen in the presence of both magnetic and electric fields for arbitrary strengths and arbitrary angles between the two fields. Up to now, only simple cases of parallel electric versus magnetic configurations have been investigated (Friedrich et al. 1994). Since in our models only crude approximations have been used, it is not possible to avoid the systematic uncertainties, especially in the low-field regime $(\leq 5 \mathrm{MG})$ where the influence of the Stark effect on the spectral lines is strong relative to the effect of Zeeman splitting. Therefore, we do not determine $\log g$ values from our spectral fits, but rather assume $\log g$ value of 8.0 for our magnetic model spectra database. This approach have been applied multiple times in the literature (Gänsicke et al. 2002; Girven et al. 2010). Additionally, we estimated the error budget introduced by the undetermined surface gravity through repeating our fitting procedure for the cases in which $\log g=7.0$ and $\log g=9.0$, and later propagating the resulting uncertainties.

The determined parameters are listed in Table 2, the errors in $T_{\text {eff }}$ are defined by the spacing of our grid points in our database. The observed spectra are compared to theoretical models in

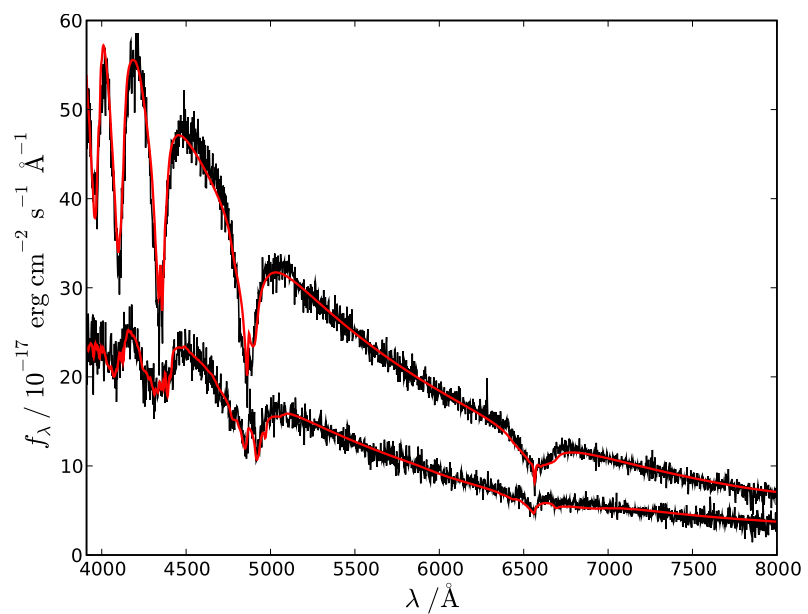

Fig. 1. Spectral fits to WD 0836+201 (top) and SDSS J0855+1640 (bottom) determined in this work. The parameters are given in Table 2. The inconsistencies in the WD $0836+201$ line fits are due to the simple treatment of the relatively large Stark effect in the low-field regime $(B \leq 5 \mathrm{MG})$.

Fig. 1. The fainter flux of SDSS J0855+1640 with respect to the flux of WD $0836+201$ despite its higher effective temperature hints a smaller radius, hence a more massive nature, if we assume both MWDs to be at the same distance.

In order to determine the MWD masses we used the synthetic magnitudes (in the ugriz photometric system of SDSS) for carbon-oxygen $(\mathrm{CO})$ core white-dwarf cooling models with thick hydrogen layer $\left(M_{\mathrm{H}} / M_{*}=10^{-4}\right)$ masses (Fontaine et al. 2001; Holberg \& Bergeron 2006) ${ }^{1}$; since these are non-magnetic models the effect of the magnetism is not included in these calculations.

$r, i$ and $z$-bands sample mostly the continuum hence these are the bands least affected by the Zeeman splitting of the Balmer lines (see Fig. 2). Moreover, in order to minimise the uncertainties of our analysis we restricted ourselves to the use of $r, i$ and $z$. If we compare the estimated MWD mass from the $i$ band with the masses derived from the $u$ band we obtain a difference as high as 8\%; in the case of masses estimated from $i$ and $g$ magnitudes, the differences are as small as $1 \%$. The results for $z$ and $r$ magnitudes are consistent within $0.05 \%$. This shows that the photometric magnitudes that correspond to spectral continuum are consistent with each other, thus we use the $i$ band in our calculations.

Given the photometric magnitudes and the estimated $T_{\text {eff }}$ of an MWD, we interpolated the synthetic magnitude grids of Holberg \& Bergeron (2006) in order to estimate its mass and cooling age. The systematic errors depend on observation errors of the photometry and the errors of $T_{\text {eff }}$ from the fits.

\footnotetext{
1 http://www . astro. umontreal .ca/ bergeron/ CoolingModels
} 
Table 2. Model fits to the SDSS observations of the objects considered in this work.

\begin{tabular}{lccccc}
\hline \hline MWD & Plate-MJD-FiberID & $B_{\text {dipole }} / \mathrm{MG}$ & $z_{\text {off }} / r_{\mathrm{WD}}$ & $i / \mathrm{deg}$ & $T_{\text {eff }} / \mathrm{K}$ \\
\hline WD 0836+201 & $2277-53705-484$ & $2.83 \pm 0.19(0.62)$ & $-0.26 \pm 0.02(0.11)$ & $26 \pm 4(33)$ & $17000 \pm 500$ \\
SDSS J0855+1640 & $2431-53818-522$ & $12.6 \pm 1.0(3.9)$ & $-0.25 \pm 0.02(0.16)$ & $44 \pm 6(40)$ & $20000 \pm 500$ \\
\hline NGC 6819-8 & - & $10.3 \pm 1.1(2.4)$ & $-0.20 \pm 0.03(0.09)$ & $52 \pm 9(22)$ & $19000 \pm 1000$ \\
\hline
\end{tabular}

Notes. The first two columns indicate the SDSS name of the object; the plate, Modified Julian Date and fiber ids of the observations; the remaining columns indicate the model fit parameters for offset dipole models. The model parameter $i$ refers to the inclination of the magnetic dipole axis with respect to the line of sight, and the offset is along the axis of the magnetic field in terms of the stellar radius. The errors in parentheses refer to the case in which $\log g$ is not fixed to the value of 8.0, but is allowed to vary between between the values of 7.0 and 9.0.

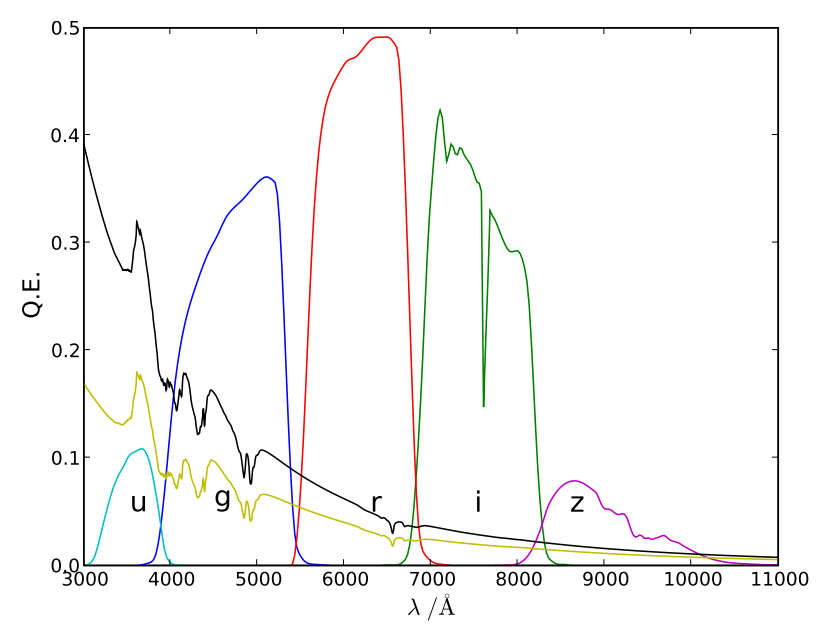

Fig. 2. The diagram above shows the response curves of the ugriz photometric pass-bands with 1.3 airmass correction applied, taken from the SDSS website ${ }^{2}$, compared to the MWD spectrum of two different effective temperatures and same magnetic field geometry which for this case is the 12.61 MG offset dipole model of SDSS J0855+1640. The fluxes are arbitrarily scaled, but consistent relative to each other. The effective temperatures are $20000 \mathrm{~K}$ (top) and $15000 \mathrm{~K}$ (bottom). The $u, g$ and $r$ bands cover the wavelengths which are effected by the magnetism in the MWD spectra for field strengths smaller than $50 \mathrm{MG}$. However this effect is minor for the $r$ band.

Furthermore, we subtracted the estimated cooling ages from the total age of the open cluster to calculate the progenitor ages of the MWDs. With this information at hand, estimating the initial masses is rather straightforward given the stellar evolution models. Hence, we interpolated the Bertelli et al. (2009) stellar evolution grids with cubic splines to calculate the progenitor masses given the progenitor lifetime, until the beginning of the Asymptotic Giant Branch phase.

\section{Results}

\section{1. $W D 0836+201$}

Previous discussions of the properties of WD $0836+201$ are mostly based on the analysis by Claver et al. (2001) where the $\pi$ components of the Zeeman splitted lines were used to determine $T_{\text {eff }}=17098 \pm 350 \mathrm{~K}$ and $\log g=8.32 \pm 0.05$. Using their values, Catalán et al. (2008a) re-estimated the mass and the progenitor mass of this object to discuss the effect of the magnetic field on IFMR. Casewell et al. (2009) mentioned that the analysis with non-magnetic model spectra may lead to uncertainties in the temperature determination. From the SDSS colors $(g-i, u-r)$

\footnotetext{
2 http://www.sdss.org/dr7/
}

of WD $0836+201$, they determined the effective temperature to be about $15000 \mathrm{~K}$, and argued on this basis that the $T_{\text {eff }}$ and $\log g$ determination from the spectroscopic non-magnetic model fits are likely to be less robust. With our theoretical spectra for magnetised white dwarf atmospheres we obtain a more reliable fit of $T_{\text {eff }}=17000 \pm 500 \mathrm{~K}$ within the spectral modeling uncertainties, a result which is consistent with Claver et al. (2001). Since the estimation of the MWD mass relies on the cluster distance of Praesepe, additional errors come from the distance errors.

There are multiple distance measurements of Praesepe. Here we adopt the value of $184.5 \pm 6 \mathrm{pc}$ as the distance to the cluster center, which was used by Casewell et al. (2009). It is the weighted mean of the HIPPARCos-based distance measurement $(m-M)_{0}=6.24 \pm 0.12$ Mermilliod et al. (1997), the groundbased parallax measurement $(m-M)_{0}=6.42 \pm 0.33($ Gatewood $\&$ de Jonge 1994) and the photometric determination $(m-M)_{0}=$ $6.33 \pm 0.04$ (An et al. 2007). It is not only a consistent approach to account for the multiple distance estimations in the literature, but also advantageous for us since we would like to compare our estimates to the results of Casewell et al. (2009).

For the determination of the progenitor ages, we relied on the cluster age of $625 \pm 50$ Myr (Perryman et al. 1998, from isochrone fits with a metallicity of $Z=0.024)$. We have chosen a value of $Z=0.027$ taken from Catalán et al. (2008a) but our mass and age determinations do only weakly depend on the choice of metallicity: If we instead use the lowest recent value for the metallicity of Praesepe ( $Z=0.019$, Claver et al. 2001; Casewell et al. 2009) the progenitor age becomes only $1 \%$ longer, which implies the progenitor mass estimates would be $0.03 M_{\odot}$ smaller, consistent with the estimates from Casewell et al. (2009).

Our results are summarized in Table 3. It should be noted that the smaller errors of the former works on WD $0836+201$ are due to underestimation of magnetic effects in spectral fits. Moreover, we can only constrain the status of WD 0836+201 as an outlier to the semi-empirical IFMR up to a limited confidence interval. Quantifying this depends strongly on the uncertainties in the $T_{\text {eff }}$ errors, which are adopted as the difference between the best fit grids in our models. However, when a greater level of confidence is applied to the observables used for the parameter estimation, the resulting values for WD $0836+201$ entail the semi-empirical IFMR, indicating no evidence for the possible effect of magnetic fields on the mass-loss. This situation is depicted in Fig. 4, where we use $3 \sigma$ errors for the photometric magnitudes and use $2000 \mathrm{~K}$ as a more realistic error for the $T_{\text {eff. }}$.

\subsection{SDSS J0855+1640}

There are certain caveats for the membership status of SDSS J0855+1640 when the rest of the Praesepe members are considered, since it is further away from the cluster center and 
Table 3. $\log g$, masses, radii and cooling ages estimated with the photometric analysis.

\begin{tabular}{lcccccc}
\hline \hline MWD & $\log g /$ dex & $M_{\mathrm{WD}} / M_{\odot}$ & Radius/0.01 $R_{\odot}$ & $t_{\text {cool }} / \mathrm{Myr}$ & $t_{\text {progenitor }} / \mathrm{Myr}$ & $M_{\mathrm{i}} / M_{\odot}$ \\
\hline WD 0836+201 & $8.33 \pm 0.08$ & $0.82 \pm 0.05$ & $1.02 \pm 0.06$ & $221_{-31}^{+37}$ & $403_{-59}^{+62}$ & $3.144_{-0.149}^{+0.181}$ \\
SDSS J0855+1640 & $8.84 \pm 0.22$ & $1.12 \pm 0.11$ & $0.67 \pm 0.14$ & $391_{-141}^{+170}$ & $234_{-150}^{+177}$ & $3.818_{-0.695}^{+1.870}$ \\
\hline NGC 6819-8 & $7.77 \pm 0.10$ & $0.50 \pm 0.05$ & $1.53 \pm 0.76$ & $66_{-10}^{+10}$ & $2430_{-250}^{+250}$ & $1.570_{-0.047}^{+0.054}$ \\
\hline
\end{tabular}

lies at the limb of the circular region defined by the tidal radius of the cluster. Within this radius the cluster members are expected to have the same kinematic properties as the cluster and high cluster proper-motions relative to the field stars enable us to discriminate the members.

The value for the tidal radius of Praesepe differs in the literature, and is increasing as the studies become more recent. Starting with 12 pc (Mermilliod et al. 1990), a higher value of 16 pc was obtained by Adams et al. (2001), and later an even higher value of $17.1 \pm 1.2 \mathrm{pc}$ was estimated by Piskunov et al. (2007). In our work we used the latest value of $18.6 \pm 1.4 \mathrm{pc}$ value of Piskunov et al. (2008).

If we assume that SDSS J0855+1640 lies at $184.5 \mathrm{pc}$, which is the distance to the core of the cluster, its angular separation from the cluster center of $4.58^{\circ}$ translates into a spatial distance of $14.7 \mathrm{pc}$. Although numerous values exist in the literature, the distance of SDSS J0855+1640 from the core puts it safely within the tidal radius of Praesepe for all determinations except the one estimated by Mermilliod et al. (1990).

Another support for the Praesepe membership of SDSS J0855+1640 is the determined cooling ages. The value estimated through the $T_{\text {eff }}$ value of $20000 \mathrm{~K}$ (see Table 3 ) yields cooling ages smaller than the age of Praesepe. Hence the cluster membership of the SDSS J0855+1640 cannot be outruled by evolutionary arguments. However, it is still necessary to carefully consider the spatial position and the distance of SDSS J0855+1640 from the core of the cluster.

Due to the mass segregation within a cluster as time progresses, relatively massive stars $\left(>1 M_{\odot}\right)$ concentrate within the core. Since these stars evolve to white dwarfs, their population is also expected to be found within the core. This is confirmed for the known white dwarfs of Praesepe and other clusters. However, there is a selection effect since spectroscopic investigations specifically aim at the core of the cluster, due to the above reasoning.

However, it is known that the number of observed white dwarfs inside open clusters is lower than expected (Weidemann 1977; Williams 2002). The fact that white dwarfs tend to evaporate from the inner core of an open cluster was already discussed for the case of the missing white dwarfs in Hyades (Weidemann et al. 1992). In this scenario, white dwarfs may leave the cluster by gaining small velocities $\left(0.1 \mathrm{~km} \mathrm{~s}^{-1}\right)$ with respect to the cluster center. Recent observations show that there is evidence for this suggestion for the case of the Hyades open cluster (Schilbach \& Roeser 2012). The authors reason that, after the formation of white dwarfs near the cluster center, due to their lower masses they are subjected also to mass segregation and move outwards.

Another possibility for gaining a kick velocity of several $\mathrm{km} \mathrm{s}^{-1}$ is due to asymmetric mass-loss during the AGB phase (Fellhauer et al. 2003). This was tested observationally for the globular cluster NGC 6397 and found to be plausible (Davis et al. 2008). Since SDSS J0855+1640 is within the tidal radius we did not consider this case for the following assessment.
Given the cooling age of SDSS J0855+1640 of 390 Myr, a kick velocity of $0.1 \mathrm{~km} \mathrm{~s}^{-1}$ implies the distance travelled since its formation would be about $40 \mathrm{pc}$. This value is significantly larger than the tidal radius of Praesepe, and may explain the distance of SDSS J0855+1640 with respect to the cluster center. Although a kick velocity explains the distance of SDSS J0855+1640 from the cluster core, at the same time does not guarantee its radial distance to be same as the cluster distance. If the MWD has gained a kick velocity in the radial direction, it may lie considerably behind or in front of the cluster center.

If the maximum distance travelled is approximately 40 parsecs, and the distance of SDSS J0855+1640 to the center of the cluster is 14.7 parsecs, then the maximum possible unaccounted radial distance equals to 37.2 parsecs. This is two times the tidal radius if the value of 18.6 parsecs is assumed for the value of the tidal radius.

In order to estimate the maximum possible uncertainty, we calculated the progenitor properties of SDSS J0855+1640 using two tidal radii as the distance error. Within these uncertainties the inferred cooling age of SDSS J0855+1640 is smaller than the age of the cluster, hence its membership could not be outruled.

Our analysis shows that SDSS J0855+1640 is possibly a part of the rare group of ultra-massive white dwarfs which have masses higher than $1.1 M_{\odot}$. This group of objects are proposed to be final products of binary mergers and were observed numerously in EUV surveys (Bergeron et al. 1991; Marsh et al. 1997). Given the massive nature and the position on the IFMR of SDSS J0855+1640, a merger scenario could be probable. However, when a larger confidence interval is applied for calculating the errors of the initial and final mass of this star, these values would entail the semi-empirical IFMR (see Fig. 4) and a single star origin cannot be outruled. The nature of this star can only be understood conclusively, if its $T_{\text {eff }}$ errors could be constrained to a value smaller than $2000 \mathrm{~K}$ within $3 \sigma$ confidence intervals.

\subsection{NGC $6819-8$}

The spectrum of NGC 6819-8 is taken from Kalirai et al. (2008), where it was not analyzed in detail due to the faintness of the star and the low signal-to-noise. We have assumed the object to be a member, whereas in Kalirai et al. (2008) the membership assessment was made by comparing the observed $V$ magnitudes with the theoretical magnitude from fitting the Balmer lines. The comparison indicated that these magnitudes were inconsistent by more than $1 \sigma$, so the white dwarf was assumed not to be a part of the cluster. Kalirai et al. (2008) used a strict cut to ensure that only real members are considered, however we cannot employ such a method for our MWDs due to the complications involved in the modeling of the photometric magnitudes under the influence of the magnetic fields (see Sect. 3). We assumed the membership of this object, based solely on the fact that its location agrees with the WD cooling sequence of the cluster. 


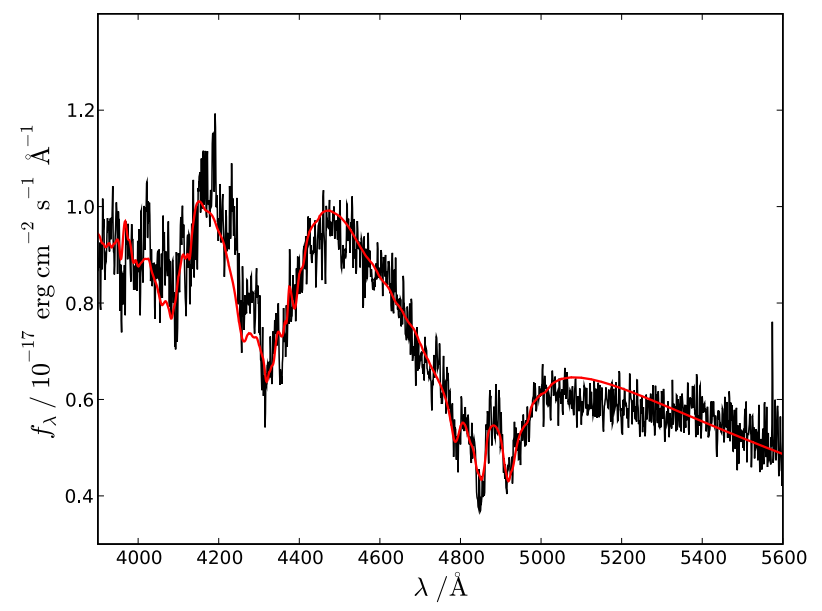

Fig. 3. Spectral fit to NGC 6819-8. Fit parameters are given in Table 2.

Our spectral analysis yielded the $T_{\text {eff }}$ and the mass of the white dwarf. Subsequently we used the same methods used for the Praesepe white dwarfs to estimate the initial mass of the object. One difference of our analysis was our usage of $V$ magnitudes rather than the $i$ band. This might introduce extra errors since the $V$ band is influenced by the splitting of hydrogen $\beta$ line. However, the estimated mass error should be smaller than $1 \%$, enumerated in the comparison between estimates with $i$ band which represents the continuum flux and the $g$ band which covers multiple Balmer lines (see Fig. 2). For the fits an additional error is introduced due to the short wavelength interval of the spectrum, which makes it harder to distinguish between models with different $T_{\text {eff. }}$.

Due to the aforementioned reasons, the main uncertainty in the (final) mass of the NGC 6819-8 comes from the inaccuracy of the $T_{\text {eff }}$ determination. However the error in the initial mass of the NGC 6819-8 is not affected by the uncertainties of the spectral modeling since the error of the cluster age dominates over other uncertainties in the spectral analysis as well as the white dwarf cooling age itself. This means that the error of the progenitor mass of the object is comparable to the errors of the progenitor masses of other objects in the cluster.

The estimation of the cluster age is achieved by theoretical isochrone fitting, which is dependent precisely on the distance, metallicity and reddenning. The distance and metallicity for the cluster NGC 6819 have been estimated through main sequence fitting by Kalirai et al. (2001). In their work the adopted reddening of $E(B-V)=0.10-0.14$ yielded slightly larger values $\left((m-M)_{v}=12.30 \pm 0.12\right)$ with respect to the previous studies. This was due to the reddening values being smaller. Using these metallicity, reddening and distance parameters, the isochrones yield 2.5 Gyr for the age of the cluster (Kalirai et al. 2001, 2008). It is noted that the degeneracy of the input parameters might induce a about 10 percent change in the result - which is the error adopted for the age in this work - however, the parameters are in agreement with the literature and, more importantly the lower main sequence were reproduced well by their model.

Using the $V$ magnitude of 23.3 for NGC $6819-8$, the distance modulus for NGC 6819 as $12.30 \pm 0.12$, and a metallicity of $Z=$ 0.017 (all parameters from Kalirai et al. 2008), we reached a progenitor mass of $1.57 M_{\odot}$ which is similar to the rest of the white dwarfs in the cluster. For comparison, we have applied our method to get the progenitor mass of the non-magnetic white dwarfs in NGC 6819, and reproduced the results of Kalirai et al. (2008).

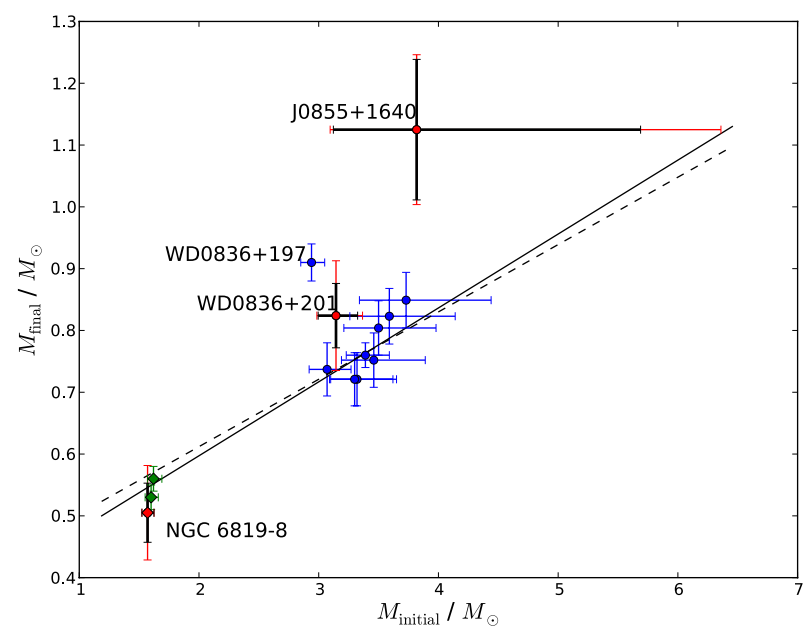

Fig. 4. IFMR and the position of the Praesepe white dwarfs (circles, Casewell et al. 2009) and NGC 6819 white dwarfs (diamonds, Kalirai et al. 2008) are shown in this diagram. The solid line is the best fit of Casewell et al. (2009), and the dashed line is the best fit from Kalirai et al. (2008) which is sensitive to the low-mass end. Red circles denote the positions of the MWDs discussed in this paper, and WD 0836+197 is a known outlier. For the MWDs considered in this work, the bold (black) errors correspond to the estimations made with the standard procedure as explained in Sect. 3, whereas the thin (red) error bars correspond to the $3 \sigma$ error estimations including an adopted $T_{\text {eff }}$ error of $2000 \mathrm{~K}$.

Given the uncertainties in the cluster age, the estimated progenitor mass of NGC 6819-8 is the most precise for the known MWD population. Although the progenitor mass is relatively low, it is still within the mass range of Ap stars, which is the progenitor population suggested by the fossil-field hypothesis.

After evaluating the errors and estimating the values of final and progenitor mass of NGC 6819-8, we end up with values comparable to the rest of the NGC 6819 white dwarfs. Within the uncertainties we conclude that there is no evidence of any effect of magnetism on the mass-loss history of NGC 6819-8.

\section{Discussion and conclusions}

In this work we investigated the evolutionary histories of MWDs which are probable members of open clusters. First of all, we correlated the proper-motions of the known magnetic DA white dwarfs in the SDSS with the open clusters. Afterwards we estimated the properties of probable cluster MWDs with our magnetic spectral modeling, and used the cluster properties to further constrain their evolutionary history. This method was applied to two currently known cluster MWDs (WD 0836+201 and NGC 6819-8) for the first time and additionally to a newly discovered SDSS J0855+1640.

We also compared our results with the the former analyses which have been undertaken with the use of non-magnetic WD spectral fits. By that, we assessed the conditions of applicability of non-magnetic analysis for MWDs. Our results show that SDSS J0855+1640 is probably an ultra-massive MWD and NGC 6819-8 has properties very similar to the rest of NGC 6819 members.

Furthermore, through the use of cluster properties we also estimated the progenitor masses of these MWDs. In Fig. 4 we compare our results and the data from Praesepe and NGC 6819 cluster white dwarfs within the scope of IFMR. Our calculations show that within the uncertainties of the magnetic analysis, all 
of the MWDs follow the general trend of the IFMR for nonmagnetic white dwarfs derived from seven open clusters and measurements from 41 white dwarfs (Casewell et al. 2009). It should also be noted that this general trend is not followed by the known outlier WD 0836+197, for which neither magnetic field nor rapid rotation was detected; it was proposed to be a possible radial velocity variable or the product of a blue straggler (Casewell et al. 2009).

The most important diagnostic tool in the estimation of the white dwarf and the progenitor masses is the determination of the effective temperature. The current progress in the magnetic spectral analysis allow for the estimating the best fit for this parameter however the formal determination of the confidence intervals is still not possible due to the complications caused by the effect of magnetism on the spectra. Hence in our work we adopt the distance between the bins in our database as errors. Furthermore, when we repeat our calculations with $3 \sigma$ errors for $T_{\text {eff }}$, we show that the the estimated progenitor and white dwarf masses would entail the semi-empirical IFMR values (see Fig. 4). According to these calculations, to be able to conclusively determine the effect of magnetism on the mass-loss, the magnetic diagnostics should be able to attain $T_{\text {eff }}$ errors smaller than $2000 \mathrm{~K}$ within $3 \sigma$ confidence intervals. In order to conclusively quantify the confidence levels of complicated $\chi^{2}$ topographies, a Markov chain Monte Carlo type of analysis is needed however, this is beyond the scope of this work.

For the the high-mass white dwarf SDSS J0855+1640 we resolve that it could also be the result of binary evolution as discussed by Bergeron et al. (1991) and Marsh et al. (1997). In this case it cannot be compared to the IFMR of single stars. The incidence of magnetism among ultra-massive white dwarfs is rather high (Vennes \& Kawka 2008), suggesting a possible relation of the evolution histories, masses and the magnetic fields.

The membership of SDSS J0855+1640 could be clarified through the determination of its trigonometric parallax e.g., with Gaia (see e.g. Torres et al. 2005; Jordan 2007). This measurement is also important for determining the origin of this star, since our mass determination is based on the assumption that the star is at the distance of the center of the Praesepe open cluster.

For the low mass end, NGC 6819-8 presents similar properties - if not a slightly lower progenitor mass - with respect to the rest of the NGC 6819 cluster members. When the position of NGC 6819-8 in the IFMR plot is compared to the solid line in Fig. 4, which is the best fit of the IFMR for the low-mass end (Kalirai et al. 2008), no considerable difference can be observed within the error bars.

One should also note that there is considerable scatter for the estimated initial masses smaller than about $3 M_{\odot}$ which was also observed for NGC 2099 (M 37, Kalirai et al. 2005). It was proposed that the theoretical uncertainties of mass-loss in this regime might also be the reason for this discrepancy. At this stage it is difficult to distinguish between the observed scatter in the low mass end due to other physical mechanisms (i.e. metallicity), and the effect of magnetism.

It is also possible to test the IFMR using double white dwarfs in wide binary systems, since these systems are assumed to have evolved without interacting. The number of known MWDs in WD-WD wide binaries is six, namely REJ 0317-853 (Barstow et al. 1995; Ferrario et al. 1997), SDSS J1300+5904 (Girven et al. 2010) and recently SDSS J092646.88+132134.5 (SDSS J0926+1321), SDSS J150746.80+520958.0 (SDSS J1507+5209, Dobbie et al. 2012), SDSS J074853.07+302543.5 and SDSS J150813.24+394504.9
(Dobbie et al. 2013). Their evolutionary status presents different challenges.

The ultra-massive and hot nature of RE J 0317-853 with respect to its non-magnetic counterpart turns out to be problematic for a single star. This problem of "age dilemma" was resolved by invoking a scenario where the system initially consists of three components and RE J 0317-853 is the result of a close binary merger (Ferrario et al. 1997). A similar evolutionary dilemma was also observed for SDSS J1507+5209, where the MWD component of the binary is relatively hot and young compared to the non-magnetic one (Dobbie et al. 2012), hence a triple system was also suggested as an explanation. The remaining MWD in wide WD-WD pairs do not show similar problems.

A similar type of analysis can be undertaken with common proper motion (CPM) binaries consisting a white dwarf and a FGK type star, where the total age and the metallicity of the white dwarf can be assessed by analyzing its companion. Catalán et al. (2008b) undertook the first of these studies in which one of the objects (40Eri B, WD 0413-017) is an MWD. Their observational data show dispersion with respect to the semi-empirical IFMR, however whether the cause of this dispersion is magnetism and/or rotation has not been discussed in detail. It should be noted that the magnetic field strength of 40 Eri $\mathrm{B}(\approx 2.3 \mathrm{kG}$, Fabrika et al. 2000) is significantly lower than the magnetic field of MWDs in this work (>1 MG).

Currently there are ten MWDs with determined white dwarf and progenitor masses. Not considering the two objects RE J 0317-853 and SDSS J1507+5209 which are believed to have formed by mergers, it has been suggested that magnetism could be a factor in modifying the mass-loss based on the discovery of WD 0836+201 (Claver et al. 2001). However our analysis, which also entails WD $0836+201$, shows that when the effect of magnetism on the observed spectrum is included in the uncertainties, the deviation of the properties of MWDs from the semiempirical IFMR disappears. The conclusive test for the extent of the dispersion caused in the IFMR by the magnetic fields will be possible through better magnetic diagnostics and more precise distance measurements.

Acknowledgements. We thank Andreas Just, Sigfried Röser and Elena Schilbach for useful discussions concerning the kinematics of open cluster members. We also thank Silvia Catalán for her useful comments on the manuscript. This work was partly supported by the DLR under grant 50 OR 0802. B.K. acknowledges support by the MICINN grant AYA08-1839/ESP, by the ESF EUROCORES Program EuroGENESIS (MICINN grant EUI2009-04170), by the 2009SGR315 of the Generalitat de Catalunya and EU-FEDER funds.

\section{References}

Adams, J. D., Stauffer, J. R., Monet, D. G., Skrutskie, M. F., \& Beichman, C. A. 2001, AJ, 121, 2053

An, D., Terndrup, D. M., Pinsonneault, M. H., et al. 2007, ApJ, 655, 233

Anand, S. P. S. 1965, Proc. Nat. Acad. Sci., 54, 23

Angel, J. R. P., Borra, E. F., \& Landstreet, J. D. 1981, ApJS, 45, 457

Barstow, M. A., Jordan, S., O’Donoghue, D., et al. 1995, MNRAS, 277, 971

Bergeron, P., Kidder, K. M., Holberg, J. B., et al. 1991, ApJ, 372, 267

Bertelli, G., Nasi, E., Girardi, L., \& Marigo, P. 2009, A\&A, 508, 355

Casewell, S. L., Dobbie, P. D., Napiwotzki, R., et al. 2009, MNRAS, 395, 1795

Catalán, S., Isern, J., García-Berro, E., \& Ribas, I. 2008a, MNRAS, 387, 1693

Catalán, S., Isern, J., García-Berro, E., et al. 2008b, A\&A, 477, 213

Claver, C. F., Liebert, J., Bergeron, P., \& Koester, D. 2001, ApJ, 563, 987

Davis, D. S., Richer, H. B., King, I. R., et al. 2008, MNRAS, 383, L20

Dobbie, P. D., Napiwotzki, R., Burleigh, M. R., et al. 2009, MNRAS, 395, 2248

Dobbie, P. D., Baxter, R., Külebi, B., et al. 2012, MNRAS, 421, 202

Dobbie, P. D., Külebi, B., Casewell, S. L., et al. 2013, MNRAS, 428, L16

Dominguez, I., Straniero, O., Tornambe, A., \& Isern, J. 1996, ApJ, 472, 783

Eggen, O. J., \& Greenstein, J. L. 1965, ApJ, 142, 925

Euchner, F., Jordan, S., Beuermann, K., Gänsicke, B. T., \& Hessman, F. V. 2002, A\&A, 390, 633 
Fabrika, S. N., Valyavin, G. G., Burlakova, T. E., Barsukova, E. A., \& Monin, D. N. 2000, in Magnetic Fields of Chemically Peculiar and Related Stars, eds. Y. V. Glagolevskij, \& I. I. Romanyuk, 218

Fellhauer, M., Lin, D. N. C., Bolte, M., Aarseth, S. J., \& Williams, K. A. 2003, ApJ, 595, L53

Ferrario, L., Vennes, S., Wickramasinghe, D. T., Bailey, J. A., \& Christian, D. J. 1997, MNRAS, 292, 205

Fontaine, G., Brassard, P., \& Bergeron, P. 2001, PASP, 113, 409

Friedrich, S., Ostreicher, R., Ruder, H., \& Zeller, G. 1994, A\&A, 282, 179

Gänsicke, B. T., Euchner, F., \& Jordan, S. 2002, A\&A, 394, 957

Gatewood, G., \& de Jonge, J. K. 1994, ApJ, 428, 166

Gianninas, A., Bergeron, P., \& Ruiz, M. T. 2011, ApJ, 743, 138

Girven, J., Gänsicke, B. T., Külebi, B., et al. 2010, MNRAS, 404, 159

Holberg, J. B., \& Bergeron, P. 2006, AJ, 132, 1221

Jordan, S. 1992, A\&A, 265, 570

Jordan, S. 2007, in 15th European Workshop on White Dwarfs, eds. R. Napiwotzki, \& M. R. Burleigh, ASP Conf. Ser., 372, 139

Jordan, S., \& Schmidt, H. 2003, in Stellar Atmosphere Modeling, eds. I. Hubeny, D. Mihalas, \& K. Werner, ASP Conf. Ser., 288, 625

Kalirai, J. S., Richer, H. B., Fahlman, G. G., et al. 2001, AJ, 122, 266

Kalirai, J. S., Richer, H. B., Reitzel, D., et al. 2005, ApJ, 618, L123

Kalirai, J. S., Bergeron, P., Hansen, B. M. S., et al. 2007, ApJ, 671, 748

Kalirai, J. S., Hansen, B. M. S., Kelson, D. D., et al. 2008, ApJ, 676, 594

Kalirai, J. S., Saul Davis, D., Richer, H. B., et al. 2009, ApJ, 705, 408

Kawka, A., Vennes, S., Schmidt, G. D., Wickramasinghe, D. T., \& Koch, R. 2007, ApJ, 654, 499

Kepler, S. O., Pelisoli, I., Jordan, S., et al. 2013, MNRAS, 429, 2934

Kharchenko, N. V., Piskunov, A. E., Röser, S., Schilbach, E., \& Scholz, R. 2005, A\&A, 438, 1163

Kleinman, S. J., Kepler, S. O., Koester, D., et al. 2013, ApJS, 204, 5

Koester, D., \& Weidemann, V. 1980, A\&A, 81, 145

Külebi, B., Jordan, S., Euchner, F., Gänsicke, B. T., \& Hirsch, H. 2009, A\&A, 506,1341

Li, N., \& Thakar, A. R. 2008, Comput. Sci. Eng., 10, 18
Liebert, J. 1988, PASP, 100, 1302

Liebert, J., Bergeron, P., \& Holberg, J. B. 2003, AJ, 125, 348

Luyten, W. J. 1962, in The Observatory, Univ. Minnesota, Minneapolis, 1953, 31,1

Marigo, P. 2001, A\&A, 370, 194

Marsh, M. C., Barstow, M. A., Buckley, D. A., et al. 1997, MNRAS, 287, 705

Mermilliod, J., Weis, E. W., Duquennoy, A., \& Mayor, M. 1990, A\&A, 235, 114

Mermilliod, J., Turon, C., Robichon, N., Arenou, F., \& Lebreton, Y. 1997, in Hipparcos - Venice '97, eds. R. M. Bonnet, E. Høg, P. L. Bernacca, et al., ESA SP, 402, 643

Munn, J. A., Monet, D. G., Levine, S. E., et al. 2004, AJ, 127, 3034

Ostriker, J. P. 1966, AJ, 71, 394

Perryman, M. A. C., Brown, A. G. A., Lebreton, Y., et al. 1998, A\&A, 331, 81

Piskunov, A. E., Schilbach, E., Kharchenko, N. V., Röser, S., \& Scholz, R. 2007, A\&A, 468, 151

Piskunov, A. E., Schilbach, E., Kharchenko, N. V., Röser, S., \& Scholz, R. 2008, A\&A, 477, 165

Portegies Zwart, S. F., McMillan, S. L. W., Hut, P., \& Makino, J. 2001, MNRAS, 321,199

Roxburgh, I. W. 1965, ZAp, 62, 134

Schilbach, E., \& Roeser, S. 2012, A\&A, 537, A129

Schmidt, G. D., Harris, H. C., Liebert, J., et al. 2003, ApJ, 595, 1101

Somerville, R. S., \& Primack, J. R. 1999, MNRAS, 310, 1087

Torres, S., García-Berro, E., Isern, J., \& Figueras, F. 2005, MNRAS, 360, 1381

van den Bergh, S., \& Tammann, G. A. 1991, ARA\&A, 29, 363

Vanlandingham, K. M., Schmidt, G. D., Eisenstein, D. J., et al. 2005, AJ, 130, 734

Vennes, S., \& Kawka, A. 2008, MNRAS, 389, 1367

Weidemann, V. 1977, A\&A, 59, 411

Weidemann, V. 2000, A\&A, 363, 647

Weidemann, V., Jordan, S., Iben, Jr., I., \& Casertano, S. 1992, AJ, 104, 1876

Wickramasinghe, D. T., \& Ferrario, L. 2005, MNRAS, 356, 1576

Williams, K. A. 2002, Ph.D. Thesis, University of California, Santa Cruz

Williams, K. A., Bolte, M., \& Koester, D. 2009, ApJ, 693, 355 